\title{
Double carbapenem regimen used as salvage therapy to treat multidrug-resistant Klebsiella pneumoniae causing ventilator-associated pneumonia
}

\begin{abstract}
Carbapenemase-producing Klebsiella pneumoniae is an emerging threat worldwide. The appropriate therapy for infections due to these multidrug-resistant pathogens is not well defined and depends upon the susceptibilities of individual isolates, and the choices are often severely limited. We report a case of a 8-year-old male child with ARDS with left-sided tubercular pleural effusion who developed ventilator-associated pneumonia due to multidrug-resistant Klebsiella pneumoniae treated successfully with a regimen comprising a combination of colistin and double carbapenem.
\end{abstract}

Key words: double carbapenem, ventilator-associated pneumonia, Klebsiella pneumoniae

Adv Respir Med. 2021; 89: 203-206

\section{Introduction}

Klebsiella pneumoniae is a frequent cause of nosocomial infections [1]. A rise of antimicrobial drug resistance in Klebsiella pneumoniae raises serious therapeutic challenges [2]. Among several mechanisms of drug resistance in Klebsiella pneumoniae, carbapenemases are increasingly recognized worldwide. They are particularly prevalent in Klebsiella pneumoniae from several geographic areas, including the Indian subcontinent and the Mediterranean countries [3]. New antibiotic options are urgently needed for the treatment of carbapenem-resistant Enterobacteriaceae infections. We report a case of a 8-year-old male child with ARDS with left-sided tubercular pleural effusion who developed ventilator-associated pneumonia due to multidrug-resistant Klebsiella pneumoniae treated successfully with a regimen comprising a combination of colistin and double carbapenem.

\section{Case report}

A 8-year-old male child on mechanical ventilation was shifted to our hospital from another hospital with a history of increasing oxygen requirement and radiological deterioration. He presented to the previous hospital with one-week history of cough, fever and breathlessness. Pleural tap was done at the previous hospital, which was positive for Mycobacterium tuberculosis on gene xpert. On presentation, the child was already on meropenem, vancomycin and antitubercular medication for the past 3 days. In spite of treatment, the general condition of the patient was deteriorating, thus he was shifted to our setup. On admission to our hospital, the patient was febrile, on mechanical ventilation with oxygen requirement of $70 \%$, end expiratory pressure of $7 \mathrm{~mm}$ $\mathrm{Hg}$ and inspiratory pressure of $26 \mathrm{~mm} \mathrm{Hg}$. $\mathrm{He}$ was generating tidal volumes of around $200 \mathrm{~mL}$. On suctioning of the endotracheal tube blood 
clots were seen. His laboratory investigations revealed raised serum bilirubin of $2.1 \mathrm{mg} / \mathrm{dL}$, aspartate aminotransferase of $313 \mathrm{U} / \mathrm{L}$, alanine aminotransferase of $667 \mathrm{U} / \mathrm{L}$, international normalized ratio -1.5 , total leukocyte count of $15 \times$ $10^{3} / \mu \mathrm{L}$ and raised C-reactive protein of $23.9 \mathrm{mg} / \mathrm{L}$. Endotracheal secretions showed the growth of Acinetobacter baumannii with colony count: $>100000$, colony-forming units $/ \mathrm{ml}$ which was sensitive to colistin, fosfomycin, minocycline and ceftriaxone EDTA sulbactum (Figure 1). The antibiotics and antitubercular therapy were changed to ceftriaxone EDTA sulbactum $1.5 \mathrm{mg} 12$ hourly, vancomycin $500 \mathrm{mg} 8$ hourly and levofloxacin $500 \mathrm{mg}$ once daily, amikacin $500 \mathrm{mg}$ once daily, ethambutol $600 \mathrm{mg}$ once daily.

There was sudden worsening of respiratory pattern on the third day of presentation with subcutaneous emphysema. Computed tomography of the chest was done (Figure 2,3), which showed bilateral patches of consolidation with crazy paving in both the lungs, along with pulmonary interstitial emphysema with extensive pneumo-mediastinum, minimal left pneumothorax and subcutaneous emphysema.

Intercostal chest drain was inserted on the left side. The child was not relieved of fever even after three days of changing antibiotics. Blood, pleural fluid and urine cultures were sterile. Bronchial lavage revealed the growth of multidrug-resistant Acinetobacter baumannii which was sensitive to colistin, ceftriaxone EDTA sulbactum and fosfomycin. Then colistin (loading dose of $6000000 \mathrm{IU}$, then 3000000 IU every 12 hours) was started, and ceftriaxone EDTA sulbactum (1.5 mg every 12 hours) was continued. As liver functions improved, isoniazid $300 \mathrm{mg}$ was started and aminoglycoside stopped due to risk of significant side effects in combination with collistin. The patient became afebrile after 48 hours of revising the antibiotics. Subsequently, rifampicin was re-introduced. After two doses of rifampicin $450 \mathrm{mg}$, the boy started having bleeding through the endotracheal tube, and repeated liver functions showed deterioration (international normalized ratio -1.8 , platelet count -110000 , serum bilirubin $-2.1 \mathrm{mg} / \mathrm{dL}$, alanine aminotransferase - $209 \mathrm{U} / \mathrm{L}$, aspartate aminotransferase- $45 \mathrm{U} / \mathrm{L}$ ). On ultrasound of the whole abdomen, hepatomegaly with minimal ascites was present. Rifampicin was stopped and fresh frozen plasma was given to control bleeding. As there was a problem with ventilation, therapeutic bronchoscopy was done to remove the clots. Bleeding tendency on re-introducing rifampicin could be due to part of sepsis

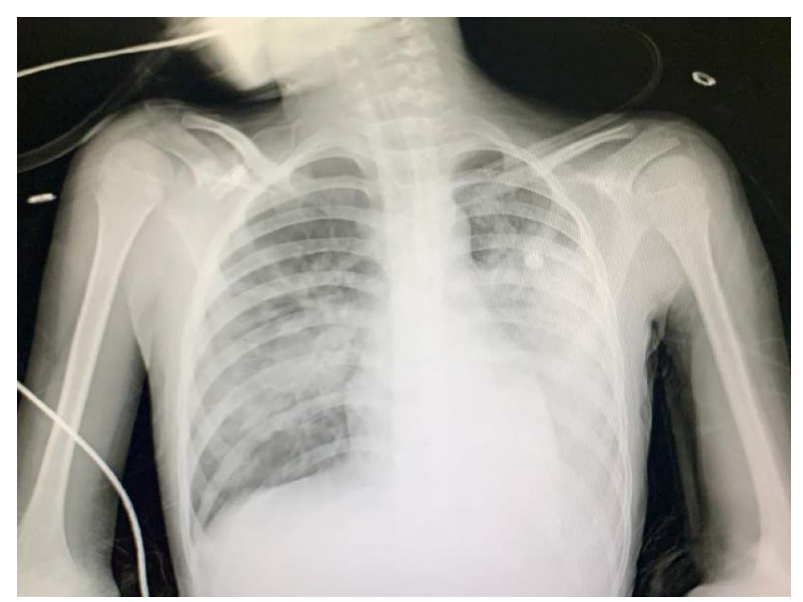

Figure 1. Chest radiography (anterior-posterior view) showing bilateral consolidation

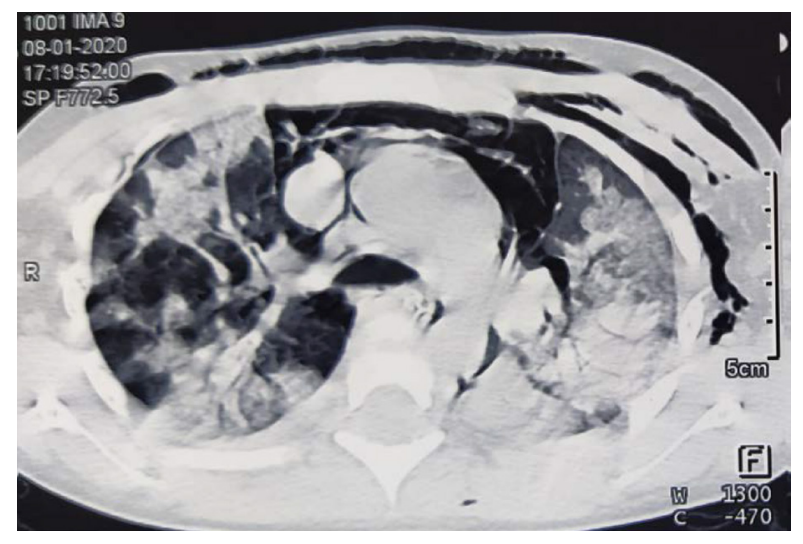

Figure 2. High resolution computed tomography of the chest showing bilateral patches of consolidation

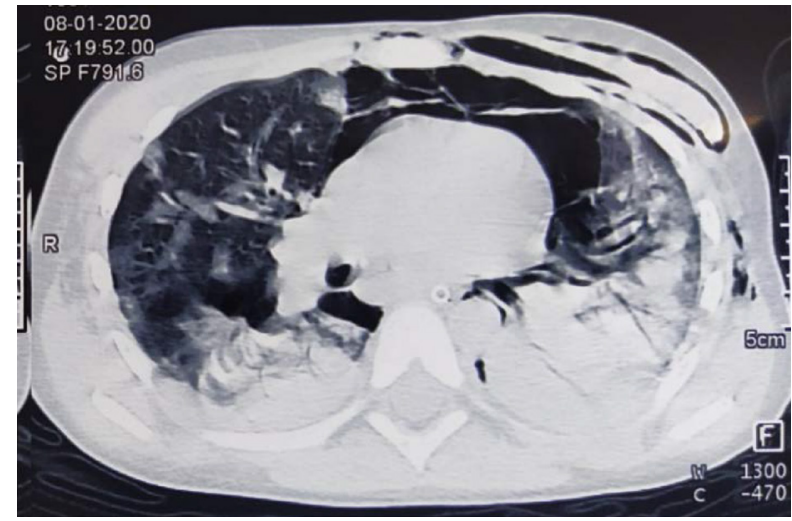

Figure 3. High resolution computed tomography of the chest showing bilateral patches of consolidation with pneumomediastinum, pneumothorax and subcutaneous emphysema

induced by disseminated intravascular coagulation or some idiosyncratic reaction. There was a gradual improvement in the patient's general condition. Percutaneous tracheostomy was done. 


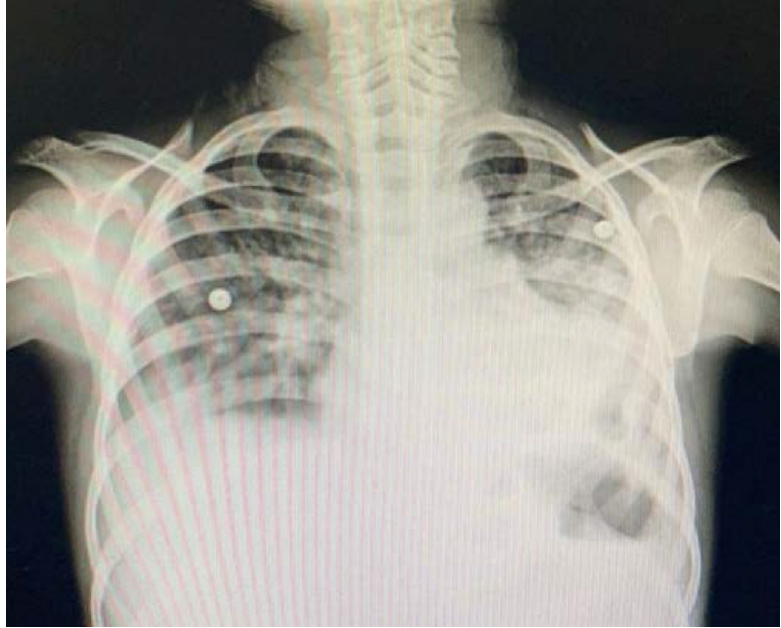

Figure 4. Chest radiography showing an increase in left lower zone infiltrates

The boy started improving clinically and radiologically. In due course, pyrazinamide $750 \mathrm{mg}$ once daily was started. Again, after 12 days of ceftriaxone EDTA sulbactum and colistin combination, the patient started having high-grade fever upto $104^{\circ} \mathrm{F}$ and oxygen requirement increased. There was worsening in inflammatory markers and on radiology (Figure 4) with an increase in left lower zone infiltrates.

Repeat tracheal aspirates showed carbapenemase-producing multidrug-resistant Klebsiella pneumoniae which was sensitive only to colistin (minimum inhibitory concentration $\leq 0.5 \mu \mathrm{g} / \mathrm{mL}$ ), which was already going on. The patient was started on combination of imipenem (MIC $\geq 16 \mu \mathrm{g} / \mathrm{mL}$ ) in extended infusion, ertapenem (MIC $\geq 8 \mu \mathrm{g} / \mathrm{mL}$ ), and colistin was continued. Fever subsided within 48 hours of starting the combination with gradual normalization of inflammatory markers. In due course, the patient was de-cannulated. Colistin and double carbepenem regimen was continued for 14 days and the child was discharged in good clinical condition. Chest radiograph done at follow-up showed almost complete clearance of infiltrates (Figure 5). Follow-up chest radiographs showed almost complete clearance of the infiltrates.

\section{Discussion}

The increasing global prevalence of carbapenem-resistant Enterobacteriaceae (CRE) combined with the decline in effective antimicrobial therapies is a serious public healthcare problem. According to CDC, CRE is defined as Enterobacteriaceae that are resistant to any carbapenem antimicrobial (i.e., minimum inhibitory concen-

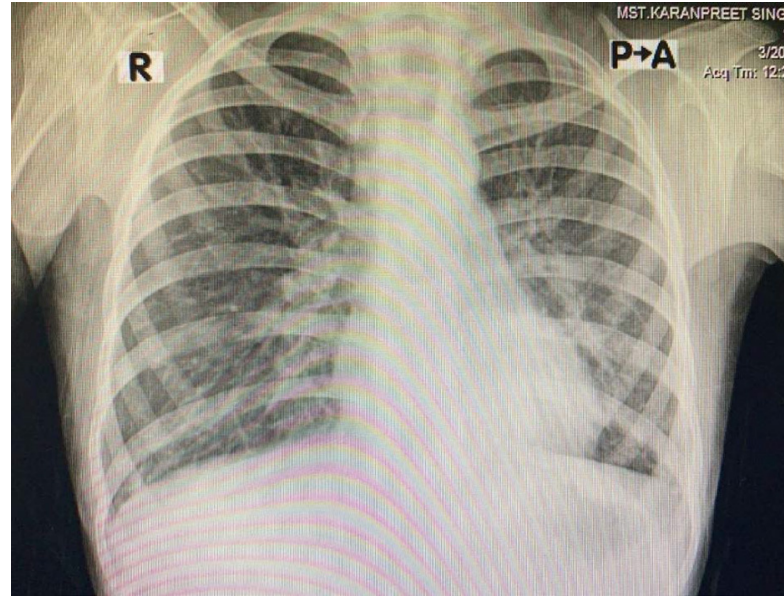

Figure 5. Follow-up chest radiography showing complete resolution of opacities

tration of $\geq 4 \mathrm{mcg} / \mathrm{mL}$ for doripenem, meropenem or imipenem OR $\geq 2 \mathrm{mcg} / \mathrm{mL}$ for ertapenem) or documented to produce carbapenemase [4]. Infections caused by these Gram-negative multidrug-resistant organisms resulted in high mortality rates, prolonged hospitalization and increased cost of care [5]. Currently, the available antibiotic options to combat these organisms are limited. So, new therapeutic approaches against these burgeoning organisms are needed. Recently, the double carbapenem regimen has been come up as a valid therapeutic option in severe infections due to pandrug-resistant Klebsiella pneumonia [6].

This case confers how the combination of colistin with ertapenem plus imipenem was effective and synergistic against a multidrug-resistant Klebsiella pneumoniae causing ventilator-associated pneumonia, even in the presence of high MIC values. The rationale for this combination has not been extensively explored, it is hypothesized that one of the carbapenem compounds distracts the carbapenemase enzyme acting as a suicide inhibitor, thus allowing and preserving the other carbapenem's activity [7]. Carbapenemase enzyme has preferential affinity for ertapenem, due to the ease of hydrolysis versus that of imipenem. Since enzyme is consumed during this interaction with ertapenem, higher concentrations of imipenem are present in the vicinity of the organism that would otherwise be recognized if copious amounts of enzyme were freely available to degrade imipenem. After that disruption caused to the outer bacterial cellular membrane by colistin allowing other drugs to reach adequate intracellular concentrations. Despite hydrolysis of carbapenems by the carbapenemase enzyme, these compounds perpetuate their bactericidal 
effect. This has been demonstrated both in vitro and in animal models [8,9].

A study was conducted to determine therapeutic strategy for pandrug-resistant Klebsiella pneumoniae severe bloodstream infection by Oliva et al.; it showed combination of colistin with ertapenem plus meropenem manifest rapid bactericidal activity, even at subinhibitory concentrations. Therefore, given the potent in vitro effect and the good clinical outcome of the patient, it suggested that colistin might be useful as an initial therapeutic add-on against pandrug-resistant organisms, rapidly decreasing the bacterial amount and limiting drug toxicity [10].

Another combination, colistin-rifampin may have a role in the treatment of multidrug-resistant Klebsiella pneumoniae and may possibly slow the selection of hetero-resistant subpopulations during colistin therapy. A study conducted to determine synergistic activity of colistin plus rifampin against colistin-resistant KPC-producing Klebsiella pneumonia by Tascini et al. showed that colistin plus rifampin is the most consistently synergistic combination against KPC-producing Klebsiella pneumoniae isolates, including colistin-resistant strains [11]. Combination is based on the principle that perturbation of the outer bacterial cellular membrane by colistin may favor the uptake of rifampin, allowing the drug to reach sufficient intracellular concentrations to inhibit protein synthesis. But in our case, we were not able to use this regimen due to rifampicin-induced hepatotoxicity and bleeding tendencies.

A source of these multidrug-resistant organisms could be a prolonged stay in hospital, and in addition, on mechanical ventilation. We treated our patient with colistin and double carbapenem regimen for 14 days, but we stopped colistin 6 days before the completion of regimen as the patient was already on colistin for the previous 8 days and he recovered clinically as well as radiologically.

\section{Conclusions}

In conclusion, this case indicates that this regimen is a valid and effective therapeutic strategy in treating severe infections caused by carbapenemase-producing Klebsiella pneumonia. Contact precautions and active surveillance are common measures that should be employed for controlling the spread of these microorganisms in hospitals.

\section{Conflict of interest}

None declared.

\section{References:}

1. Podschun R, Ullmann U. Klebsiella spp. as nosocomial pathogens: epidemiology, taxonomy, typing methods, and pathogenicity factors. Clin Microbiol Rev. 1998; 11(4): 589-603, indexed in Pubmed: $\underline{9767057}$.

2. Rice LB. Federal funding for the study of antimicrobial resistance in nosocomial pathogens: no ESKAPE. J Infect Dis. 2008; 197(8): 1079-1081, doi: 10.1086/533452, indexed in Pubmed: 18419525.

3. Nordmann P, Poirel L. The difficult-to-control spread of carbapenemase producers among Enterobacteriaceae worldwide. Clin Microbiol Infect. 2014; 20(9): 821-830, doi: 10.1111/14690691.12719, indexed in Pubmed: 24930781.

4. Facility guidance for control of carbapenem-resistant Enterobacteriaceae (CRE)-November 2015 update CRE toolkit.

5. Holmberg SD, Solomon SL, Blake PA. Health and economic impacts of antimicrobial resistance. Rev Infect Dis. 1987; 9(6): 1065-1078, doi: 10.1093/clinids/9.6.1065, indexed in Pubmed: 3321356.

6. Oliva A, D'Abramo A, D'Agostino C, et al. Synergistic activity and effectiveness of a double-carbapenem regimen in pandrug-resistant Klebsiella pneumoniae bloodstream infections. J Antimicrob Chemother. 2014; 69(6): 1718-1720, doi: 10.1093/ jac/dku027, indexed in Pubmed: 24521856.

7. Camargo JF, Simkins J, Beduschi T, et al. Successful treatment of carbapenemase-producing pandrug-resistant Klebsiella pneumoniae bacteremia. Antimicrob Agents Chemother. 2015; 59(10): 5903-5908, doi: 10.1128/AAC.00655-15, indexed in Pubmed: 26386029.

8. Tzouvelekis LS, Markogiannakis A, Piperaki E, et al. Treating infections caused by carbapenemase-producing Enterobacteriaceae. Clin Microbiol Infect. 2014; 20(9): 862-872, doi: 10.1111/1469-0691.12697, indexed in Pubmed: 24890393.

9. Daikos GL, Panagiotakopoulou A, Tzelepi E, et al. Activity of imipenem against VIM-1 metallo-beta-lactamase-producing Klebsiella pneumoniae in the murine thigh infection model. Clin Microbiol Infect. 2007; 13(2): 202-205, doi: 10.1111/j.14690691.2006.01590.x, indexed in Pubmed: 17328735.

10. Oliva A, Mascellino MT, Cipolla A, et al. Therapeutic strategy for pandrug-resistant Klebsiella pneumoniae severe infections: short-course treatment with colistin increases the in vivo and in vitro activity of double carbapenem regimen. Int J Infect Dis. 2015; 33: 132-134, doi: 10.1016/j.ijid.2015.01.011, indexed in Pubmed: 25597275.

11. Tascini C, Tagliaferri E, Giani T, et al. Synergistic activity of colistin plus rifampin against colistin-resistant KPC-producing Klebsiella pneumoniae. Antimicrob Agents Chemother. 2013; 57(8): 3990-3993, doi: 10.1128/AAC.00179-13, indexed in Pubmed: 23752510. 